\section{Diagnóstico bacteriológico de la tuberculosis en Argentina: resultados de una encuesta nacional}

\author{
Bacteriological diagnosis of tuberculosis in \\ Argentina: results of a national survey
}

\footnotetext{
${ }^{1}$ Instituto Nacional de Enfermedades Respiratorias Dr. Emilio Coni, Santa Fe, Argentina.

2 Otros miembros listados al final del artículo.

Correspondencia M. S. Imaz

Instituto Nacional de Enfermedades Respiratorias Dr. Emilio Coni. Av. Blas Parera 8260, Santa Fe, Argentina.

suimaz@yahoo.com
}

\begin{abstract}
In order to assess laboratory resources, practices, and biosafety measures during mycobacterial testing and determine the usefulness and scope of mycobacterial techniques, a cross-sectional survey of public laboratories was conducted in Argentina using a structured questionnaire. Sputum smear analysis showed that both smear and culture testing centers are being provided at or above the WHO recommended density for such facilities. The microscopy quality assessment program covered most high-demand laboratories. Mean number of sputum smears per patient was low (1.6). The use of inadequate personal respiratory protection was identified in $25 \%$ of culture laboratories. Jurisdictions that cultured a higher proportion of their smear-tested sputa identified a higher proportion of smear-negative cases. The percentage of smear negatives among all bacteriologically confirmed pulmonary cases was $18.9 \%$, while most extrapulmonary cases were confirmed by culture (71.3\%). In conclusion, increasing the number of sputa studied by microscopy and culture (while expanding the coverage of the quality assessment program) is a priority; adequate respiratory protection and regular medical evaluation of laboratory staff is still needed in some laboratories.
\end{abstract}

Tuberculosis; Laboratory Techniques and Procedures; Data Collection
María Susana Imaz 1

María Delfina Sequeira 1

Red de Laboratorios Provinciales de

Tuberculosis en Argentina 2

\section{Introducción}

En Argentina, durante las últimas dos décadas, la tasa de notificación de casos de tuberculosis disminuyó desde 60,5 a 31,9 casos por 100.000 habitantes, informándose durante el año 2003, un total 12.257 casos 1. Sin embargo, las estimaciones realizadas por la Organización Mundial de la Salud (OMS) para el año 2002 indicaban que la tasa de detección de casos nuevos era sólo cercana al $65 \% 2$. En este sentido, el principal objetivo del Programa Nacional de Control de Tuberculosis (PNCTB) en Argentina, conducido por el Instituto Nacional de Enfermedades Respiratorias Dr. Emilio Coni (INER), Santa Fe, es diagnosticar los pacientes infecciosos en un estadio temprano de la enfermedad y tratarlos efectivamente, utilizando la estrategia de tratamiento acortado estrictamente supervisado que se encuentra en proceso de expansión. Durante el año 2002, el porcentaje de casos bajo tratamiento directamente observado alcanzó al 58\%, mientras que la tasa de éxito de los tratamientos llegó al 75,1\% ${ }^{3}$.

Los cinco elementos de la estrategia tratamiento acortado estrictamente supervisado, considerada como esencial para el control mundial de la tuberculosis, son: compromiso político, detección de casos usando la microscopía de esputo entre las personas que buscan la atención por tos prolongada, quimioterapia de corta duración estandarizada en condiciones adecuadas del manejo de casos, incluido el tratamiento 
directamente observado, suministro regular de medicamentos y un sistema de registro y notificación estandarizado, que permita la evaluación individual de los pacientes, así como el rendimiento general del programa ${ }^{4}$. Por tanto, uno de los elementos críticos para la implantación y expansión exitosa de la estrategia tratamiento acortado estrictamente supervisado en un país es la presencia de laboratorios adecuados 5,6. Los expertos de OMS han realizado múltiples recomendaciones en relación a la organización de los laboratorios de tuberculosis en países con medianos o bajos recursos 7 . Por otro lado, el incremento de los casos de tuberculosis asociados al virus de la inmunodeficiencia humana (VIH), agravado por la emergencia de polifarmacorresistencia ha obligado a una revisión de las normas de bioseguridad en los laboratorios de tuberculosis.

Este trabajo describe los resultados de una encuesta transversal a los laboratorios del sector público que se llevó a cabo en Argentina con el fin de determinar si la organización y funcionamiento de los laboratorios se adaptaba a las necesidades de detección de casos del PNCTB. Los propósitos específicos de este trabajo fueron: primero, obtener información actualizada acerca de la disponibilidad de servicios de laboratorios de tuberculosis y del volumen real de pruebas diagnósticas que realizaban; segundo, evaluar las prácticas de laboratorio, incluyendo medidas de bioseguridad, y tercero, determinar la utilidad y alcance de las técnicas empleadas en bacteriología de la tuberculosis.

En Argentina, los laboratorios de tuberculosis del sector público están organizados en tres niveles: (i) periférico, representado por laboratorios de diferente complejidad técnica responsables del diagnóstico bacteriológico de la enfermedad; están ubicados en centros de atención primaria y en hospitales de las 24 provincias del país, (ii) regional, representado por los Laboratorios Centrales de tuberculosis de Provincias, ubicados en las capitales de las 24 provincias; además de las funciones técnicas son responsables de planificar, financiar y supervisar los laboratorios de sus redes provinciales, y (iii) nacional, representado por dos laboratorios de Referencia, ubicados en el Instituto Nacional de Enfermedades Infecciosas (INEI, Buenos Aires) y el INER, responsables de la conducción de la red nacional. Los niveles de conducción regional y nacional de las redes de laboratorios de tuberculosis trabajan en coordinación con los respectivos niveles de los programas de control de tuberculosis.

\section{Métodos}

La información se recabó a través de un cuestionario estructurado que fue enviado a los responsables de los Laboratorios Centrales de Tuberculosis de Provincias para su distribución en los laboratorios del sector público. El contenido de la encuesta incluía preguntas acerca de:

a) Métodos empleados para el examen microscópico directo de frotis en la detección de bacilos ácido-alcohol resistentes, para el cultivo de micobacterias y para la prueba de sensibilidad a medicamentos. Medidas de bioseguridad existentes. Participación en programas de evaluación externa de calidad de microscopía.

b) Carga de trabajo de microscopía de muestras pulmonares, número de pacientes de los que se obtuvieron dichas muestras respiratorias (pacientes sintomáticos respiratorios) y volumen de muestras procesadas por cultivo.

c) Datos de identificación, tipo de muestra, resultado del examen microscópico directo y cultivo de los casos nuevos con resultado bacteriológico positivo para tuberculosis. Se solicitó la información de los resultados bacteriológicos de la primera muestra de cada paciente que cronológicamente dio lugar a un resultado positivo. Cuando un mismo paciente era informado por dos laboratorios, se consideró la información proveniente del servicio que hizo primero el diagnóstico. Los datos de identificación de los pacientes informados por los laboratorios se compararon con los de las bases nacionales de notificación de casos (disponibles en el INER), a fin de determinar si se trataba de un caso nuevo o de un paciente en tratamiento. Para los casos que no fueron encontrados en estas bases, se recurrió a la información del paciente a nivel local para la constatación de la categoría del caso.

El estudio se llevó a cabo entre febrero y septiembre de 2004, con preguntas acerca de condiciones y prácticas de laboratorio durante el período del 1 1ro de septiembre al 31 de diciembre de 2003. Los laboratorios que no respondían fueron contactados telefónicamente para tener certeza acerca de la recepción del cuestionario y para alentarlos a participar en el estudio. Para el cálculo de las tasas y proporciones basadas en estimaciones de población se utilizaron las cifras del Censo 2001, actualizadas al año 2003. Para el manejo y análisis de datos se empleó el programa Epi Info 6.04 (Centers for Disease Control and Prevention, Atlanta, Estados Unidos). Se utilizó el coeficiente de correlación de Pearson para estudiar la correlación entre grupos de datos. Con las variables que en el análisis univariado resultaron estadísticamente significativas $(\mathrm{p}<0,05)$ se desarrolló un modelo de regresión multivariado 8 . 
El número de datos incluido en cada análisis varía, debido a que no todos los laboratorios respondieron todas las preguntas.

\section{Resultados}

\section{Cobertura}

De los 724 laboratorios de tuberculosis contactados por los responsables de las redes provinciales, 519 (72\%) participaron en el estudio. Entre los laboratorios que realizaban cultivo, la cobertura alcanzó al 78\% (79/101). La respuesta fue variable por provincia. De las 24 jurisdicciones, en 18 la información provino de entre el $80-100 \%$ de sus servicios (en 12 provincias, del 100\%), en 2 de entre el $50-80 \%$ y en las restantes 4 la cobertura fue entre el 10 y $20 \%$. Globalmente, considerando la información del censo de población, se podría estimar que los laboratorios participantes cubrían el $90 \%$ de la población del país.

Además, en base a la información recolectada rutinariamente por la Red Nacional de Laboratorios de Tuberculosis, 177 (91\%) de los 195 laboratorios que no respondieron a la encuesta realizaban $<250$ frotis/año, en tanto que todos los laboratorios de cultivo que no participaron (22 de 101) procesaban < 20 muestras por semana.

\section{Disponibilidad de laboratorios}

Durante el año 2003, la Red Nacional de Laboratorios de Tuberculosis estaba compuesta por 724 servicios que realizaban examen microscópico directo. El promedio de servicios fue de 1,89/100.000 habitantes, con una variación desde 0,90 a 6,8 entre las 24 provincias.

Existían 101 servicios públicos que realizaban cultivo para micobacterias. El promedio de laboratorios de cultivo fue de 2,63 por 1.000 .000 habitantes. En una pequeña provincia del país $(0,8 \%$ de la población de Argentina), el cultivo estaba temporalmente suspendido, debido a un desperfecto en el autoclave. Excluyendo esta jurisdicción, el promedio de servicios de cultivo varió entre 0,94 y 9,34 por 1.000 .000 habitantes entre las distintas provincias. Diecinueve de estos laboratorios realizaban pruebas de susceptibilidad a drogas (promedio de 4,95 servicios/10.000.000 habitantes).

\section{Carga de trabajo}

\section{- Examen microscópico directo para la detección de bacilos ácido-alcohol resistentes}

Globalmente, durante todo el período del estudio, el número de frotis examinados fue de 66.934. En total, de los 519 laboratorios que informaron, 303 (58\%) realizaban $\leq$ cinco exámenes microscópicos directos por semana o $\leq 250$ por año, 87 (17\%) realizaban 250-499 por año, 59 (11\%) realizaban $500-999$ por año y 70 (13\%) realizaban $\geq 20$ por semana $0 \geq 1.000$ por año.

Los laboratorios participantes enviaron información no sólo acerca del volumen de muestras respiratorias estudiadas por examen microscópico directo, sino también acerca del número de pacientes sintomáticos respiratorios de los que se obtuvieron dichas muestras. El promedio de examen microscópico directo/pacientes sintomáticos respiratorios fue de 1,6 , con un rango de 1,1 a 2 entre las distintas provincias. La relación del número de pacientes investigados para encontrar un caso examen microscópico directopositivo (tasa de positividad) varió desde 147:1 a 16:1. Notablemente, esta relación no se correlacionó con la tasa de notificación de casos en las diferentes provincias. Globalmente, el promedio fue de 26:1.

\section{- Cultivo de Mycobacterium tuberculosis}

Los laboratorios participantes (79) informaron que habían cultivado 23.855 muestras (19.716 pulmonares y 4.139 extrapulmonares). Globalmente, 38 (48\%) servicios cultivaban $<20$ muestras/semana.

Tomando en cuenta la información de 12 provincias, en las cuales la cobertura del volumen de examen microscópico directo y cultivos fue $100 \%$, la relación promedio entre el número de examen microscópico directo y de cultivos realizados a muestras pulmonares fue 4 (rango 3-23 en las distintas provincias).

\section{Métodos empleados}

Casi todos los laboratorios (722/724) utilizaban el método de tinción de Ziehl-Neelsen.

Todos los servicios empleaban medios sólidos preparados con huevo; de los 79 laboratorios que participaron, 73 (que realizaban 99,2\% del volumen total de cultivos) utilizaban medios sólidos preparados caseramente por alguno de los laboratorios de la red. De acuerdo a la información recolectada, 59 de los 77 (76,6\%) laboratorios que respondieron cultivaban al menos dos muestras respiratorias por paciente. En relación al uso del 
cultivo, 54 de los 78 laboratorios que respondieron $(69,2 \%)$ utilizaban el cultivo selectivamente de acuerdo a las prioridades impartidas en las Guías de la red nacional de laboratorios de tuberculosis 9,10,11, mientras que los restantes servicios empleaban esta técnica para todo tipo de muestra. En la Tabla 1 se presenta el resto de la información referida a la estructura, técnicas y métodos empleados.

Tabla 1

Estructura, técnicas y métodos usados para el examen microscópico directo, cultivo de micobacterias y pruebas de sensibilidad a drogas en la red de laboratorios de tuberculosis de Argentina, septiembre-diciembre 2003.

\begin{tabular}{|c|c|}
\hline Método & Laboratorios \\
\hline \multicolumn{2}{|l|}{ Tinción para el examen microscópico $(\mathrm{N}=724)$ * } \\
\hline Ziehl-Neelsen & 722 \\
\hline Fluorocromo & 2 \\
\hline \multicolumn{2}{|l|}{ Cultivo de micobacterias $(\mathrm{N}=101)$ * } \\
\hline \multicolumn{2}{|l|}{ Medio de cultivo * } \\
\hline Sólido ** & 89 \\
\hline Sólido + radiométrico & 7 \\
\hline Sólido + no-radiométrico *** & 5 \\
\hline \multicolumn{2}{|l|}{ Decontaminación } \\
\hline $\mathrm{NaOH} 4 \% \mathrm{v} / \mathrm{v}$ & 71 \\
\hline NALC-NaOH standard 39 & 4 \\
\hline LSS-NaOH 40 & 1 \\
\hline Ni-informado & 25 \\
\hline \multicolumn{2}{|l|}{ Métodos usados para el cultivo en medio sólido } \\
\hline Usando una centrífuga 10,11 & 50 \\
\hline Sin el uso de centrífuga 13 & 26 \\
\hline No-informado & 25 \\
\hline \multicolumn{2}{|l|}{ Origen del medio sólido } \\
\hline Preparado en un laboratorio de la red & 73 \\
\hline Adquirido comercialmente & 6 \\
\hline No-informado & 22 \\
\hline \multicolumn{2}{|l|}{ Prueba de sensibilidad a drogas $(N=19)$} \\
\hline Método de las proporciones (medio con huevo) & 13 \\
\hline Método de proporciones + radiométrico & 4 \\
\hline Sólo radiométrico & 2 \\
\hline
\end{tabular}

* La cobertura de estos ítems es 100\% debido a que, para los servicios que no respondieron, la información fue colectada por teléfono;

** Setenta por ciento de los laboratorios usan medio de Stonebrink y LowensteinJensen. Los restantes laboratorios emplean sólo medio Lowensterin-Jensen;

*** Las pruebas no-radiométricas usadas son sistema Bactec 960 (1 laboratorio), sistema MB-Bact (1 laboratorio), Sistema Mycobacteria Growth Indicator Tube (M-GIT) (3 laboratorios).

NALC: N-acetil L-cisteina; LSS: lauril sulfato de sodio.
Barreras respiratorias en
los laboratorios de cultivo

De los 79 laboratorios que respondieron, 31(39\%) tenían una o más cabinas de seguridad biológica. Entre los restantes 48 servicios sin cabina, sólo $37(77 \%)$ reportaron que su personal utilizaba máscaras mientras realizaba procedimientos de laboratorio; sin embargo, el $24 \%$ de ellos (9 laboratorios) usaban máscaras de cirugía, que no proveen adecuada protección. Entre los 227 trabajadores de laboratorios de cultivo, nueve (4\%) tenían historia de tuberculosis durante los últimos 10 años (Tabla 2).

\section{Control de calidad externo de examen microscópico directo}

El control de calidad externo de baciloscopias, instaurado por la Red Nacional de Laboratorios de Tuberculosis, consiste en la relectura a ciegas de láminas bajo la modalidad periferia-centro 12 . Esta modalidad se basa en el envío por parte del laboratorio supervisado de todos los extendidos coloreados durante su trabajo de rutina en un período dado, a un laboratorio de referencia nacional o provincial, en el que se realiza la relectura. El laboratorio de referencia nacional es responsable de evaluar la calidad de los exámenes microscópicos directos de los laboratorios de referencia provincial, mientras que los laboratorios de referencia provincial supervisan los laboratorios intermedios y locales. Durante 2003, de los 724 laboratorios, 226 (31\%) ubicados en 23 provincias tomaron parte del programa de control de calidad; más de la tercera parte de los participantes realizaban $\geq 500$ frotis por año, por lo que tomando en cuenta el volumen de examen microscópico directo, realizado en los diferentes servicios, se podría estimar que alrededor del $70 \%$ de los exámenes microscópicos directos se realizan en laboratorios que participan en el programa. Se releyeron 5.342 láminas y la concordancia de la relectura alcanzó al 98,5\%. El $90 \%$ de las discordancias se encontraron en laboratorios periféricos de cinco provincias del país. El 30\% de los falsos positivos se encontraron en láminas que presentaban defectos en la coloración (decoloración inadecuada o presencia de cristales de fucsina).

\section{Resultados bacteriológicos}

\section{- Casos pulmonares}

Durante el período del estudio se confirmaron bacteriológicamente 2.043 casos nuevos de tuberculosis pulmonar (Tabla 3). En 59 casos la información acerca del resultado del examen 
microscópico directo no estuvo disponible, ya que dichos casos fueron informados por laboratorios de cultivo, a partir de muestras derivadas por otros servicios en los que se había realizado el examen microscópico directo. De los 1984 casos restantes, las muestras respiratorias fueron examen microscópico directo positivo en 1609 casos $(81,1 \%)$ y sólo cultivo positivo en 375 casos (18,9\%) (rango $0-42,3 \%$ en las distintas provincias).

Con el fin de identificar factores asociados al nivel de identificación de casos sólo cultivo positivo, tomamos en cuenta la información obtenida de 12 jurisdicciones en las cuales la cobertura del estudio fue $100 \%$. Se analizó el nivel de asociación entre el porcentaje sólo cultivo positivo alcanzado en cada provincia y variables relacionadas con la disponibilidad de servicios de cultivo en cada jurisdicción (número de habitantes cubiertos/laboratorio de cultivo; superficie cubierta - en $\mathrm{km}^{2}$ /laboratorio de cultivo), con el grado de utilización del cultivo (porcentaje de muestras respiratorias cultivadas en cada jurisdicción, provenientes de servicios que no realizan cultivo; porcentaje de muestras respiratorias cultivadas en relación a aquellas procesadas por examen microscópico directo) y con los métodos de cultivo empleados 10,11,13 (porcentaje de muestras cultivadas utilizando un método que involucrara uso de centrífuga) (Tabla 4). Otras variables asociadas al uso y métodos de cultivo, como el porcentaje de muestras cultivadas en laboratorios que disponen de métodos de cultivo basados en medios líquidos o que cultivan más de una muestra/paciente, no fueron analizadas, ya que en un análisis preliminar se evidenció que en este estudio no estaban estadísticamente asociadas al rendimiento individual de los laboratorios de cultivo (datos no mostrados). Sólo la relación entre el número de habitantes cubier-
Tabla 2

Barreras respiratorias utilizadas en los laboratorios que realizan cultivo para micobacterias de la red nacional de laboratorios públicos.

Argentina, septiembre-diciembre 2003.

\begin{tabular}{lcc}
\hline Práctica de bioseguridad & \multicolumn{2}{c}{ Laboratorios $(\mathbf{N}=\mathbf{7 9})$} \\
& $\mathbf{n}$ & $\%$ \\
\hline Barrera de protección respiratoria & & \\
Cabina de bioseguridad & 12 & 15,20 \\
Cabina de bioseguridad + mascarilla N95 & 19 & 24,00 \\
Mascarilla N95 & 28 & 35,40 \\
Máscara de cirugía & 9 & 11,40 \\
Ninguna barrera & 11 & 13,90 \\
Evaluación médica regular provista para los & & \\
trabajadores de laboratorio & & 30,38 \\
Sí & 24 & 69,62 \\
No & 55 & \\
\hline
\end{tabular}

tos/laboratorio y la proporción de muestras respiratorias cultivadas, en relación a aquellas procesadas por examen microscópico directo, se correlacionaron con el nivel de sólo cultivo positivo provincial $(\mathrm{r}=0,6$; $\mathrm{p}<0.05$ y $\mathrm{r}=0,74 ; \mathrm{p}<$ 0,01 , respectivamente, coeficiente de correlación de Pearson). Sin embargo, cuando realizamos un análisis de regresión múltiple, utilizando como variable dependiente el nivel provincial de casos sólo cultivo positivo, sólo la proporción de muestras respiratorias cultivadas en relación a aquellas procesadas por examen directo persistió en el modelo con un $\mathrm{r}^{2}$ de 0,59 .

La información de los resultados bacteriológicos de los pacientes de acuerdo al tipo de muestra estudiada se presenta en la Tabla 3. En muestras como el lavado broncoalveolar o lava-

Resultados bacteriológicos de los pacientes con tuberculosis pulmonar de acuerdo al tipo de muestra. Argentina, septiembre-diciembre 2003.

\begin{tabular}{|c|c|c|c|c|c|c|c|c|c|c|c|c|c|}
\hline \multirow[t]{2}{*}{ Muestra } & \multirow[t]{2}{*}{$\mathbf{N}$} & \multicolumn{2}{|c|}{$\mathrm{ED}+\mathrm{C}+$} & \multicolumn{2}{|c|}{$\mathrm{ED}+\mathrm{Cnr}$} & \multicolumn{2}{|c|}{$E D+C-$} & \multicolumn{2}{|c|}{ ED+ Ccont } & \multicolumn{2}{|c|}{ ED- $\mathrm{C}+$} & \multicolumn{2}{|c|}{ EDni C+ * } \\
\hline & & $\mathbf{n}$ & $\%$ & $\mathbf{n}$ & $\%$ & $\mathbf{n}$ & $\%$ & $\mathrm{n}$ & $\%$ & $\mathbf{n}$ & $\%$ & $\mathrm{n}$ & $\%$ \\
\hline Esputo & 1.922 & 668 & 34,7 & 833 & 43,3 & 31 & 1,6 & 11 & 0,6 & 321 & 16,7 & 58 & 3,0 \\
\hline Lavado broncoalveolar & 27 & 9 & 33,3 & 3 & 11,1 & 0 & 0,0 & 0 & 0,0 & 14 & 51,8 & 1 & 3,7 \\
\hline Lavado gástrico & 94 & 43 & 45,7 & 11 & 11,7 & 0 & 0,0 & 0 & 0,0 & 40 & 42,6 & 0 & 0,0 \\
\hline Total de casos & 2.043 & 720 & 35,2 & 847 & 41,5 & 31 & 1,5 & 11 & 0,5 & 375 & 18,3 & 59 & 2,9 \\
\hline
\end{tabular}

ED+: examen directo positivo; ED-: examen directo negativo; EDni: examen directo no informado; C+: cultivo positivo; C-: cultivo negativo; Ccont: cultivo contaminado; Cnr: cultivo no realizado.

* Casos informados por laboratorios de cultivo que recibieron las muestras de servicios en los que ya se habían realizado los exámenes directos. 
do gástrico, el porcentaje de casos sólo cultivo positivo fue alto, muy superior al encontrado en muestras de esputo. Tres de 27 lavado gástrico y 11 de 94 lavado broncoalveolar que fueron informados como examen microscópico directo-positivo, no fueron remitidos a los correspondientes laboratorios para su cultivo.

\section{- Casos extrapulmonares}

Se confirmó el diagnóstico de tuberculosis extrapulmonar en 209 casos. La Tabla 5 presenta el rendimiento global de las pruebas bacteriológicas de acuerdo a la muestra estudiada. La mayor proporción de muestras positivas fue encontrada en localizaciones pleurales, linfáticas y meníngeas.
Ciento cincuenta y dos $(72,7 \%)$ de los 209 casos se confirmaron sólo por cultivo. En nueve (4,3\%) muestras, no se realizó examen microscópico directo; éstas correspondían a muestras de sangre y médula ósea, en las que la posibilidad de obtener un resultado positivo por examen microscópico directo es muy baja. La mayoría de las muestras con examen microscópico directopositivo y cultivo-no realizado correspondieron a orina y ganglios linfáticos. Las muestras con examen microscópico directo-positivo y cultivonegativo eran materiales de biopsia, en los cuales el uso de formaldehído o sustancias antisépticas pudo haber sido responsable de la muerte de los bacilos.

Tabla 4

Coeficiente de correlación entre la proporción de casos pulmonares bacteriológicamente confirmados sólo por cultivo, obtenido en cada provincia y diferentes variables de acceso y utilización de la técnica de cultivo, Argentina, septiembre-diciembre 2003.

Sólo por cultivo

Relación de habitantes cubiertos en cada provincia/laboratorio de cultivo 0,60 *

Relación de superficie cubierta (en $\mathrm{km}^{2}$ )en cada provincia/laboratorio de cultivo

Porcentaje de las muestras respiratorias cultivadas en cada jurisdicción derivadas de servicios que no realizan cultivo

Relación de muestras procesadas por examen microscópico directo/muestras cultivadas

0,74 **

Porcentaje de muestras cultivadas utilizando un método de cultivo que involucrara el uso de centrífuga

$* p<0,05$

** $\mathrm{p}<0,01$ obtenidas mediante el coeficiente de correlación de Pearson.

Resultados bacteriológicos de 209 pacientes con tuberculosis extrapulmonar de acuerdo con el tipo de muestra. Argentina, septiembre-diciembre 2003.

\begin{tabular}{|c|c|c|c|c|c|c|c|c|c|c|c|c|c|}
\hline \multirow[t]{2}{*}{ Muestra } & \multirow[t]{2}{*}{$\mathbf{N}$} & \multicolumn{2}{|c|}{$\mathrm{ED}+\mathrm{C}+$} & \multicolumn{2}{|c|}{$\mathrm{ED}+\mathrm{Cnr}$} & \multicolumn{2}{|c|}{$E D+C-$} & \multicolumn{2}{|c|}{ ED+ Ccont } & \multicolumn{2}{|c|}{ ED- C+ } & \multicolumn{2}{|c|}{ EDnr C+ } \\
\hline & & $\mathrm{n}$ & $\%$ & $\mathbf{n}$ & $\%$ & $\mathbf{n}$ & $\%$ & n & $\%$ & $\mathbf{n}$ & $\%$ & $\mathrm{n}$ & $\%$ \\
\hline Líquido pleural & 65 & 6 & 9,2 & 0 & 0,0 & 0 & 0,0 & 0 & 0,0 & 59 & 90,8 & 0 & 0,0 \\
\hline Ganglios & 39 & 6 & 15,4 & 9 & 23,1 & 5 & 12,8 & 0 & 0,0 & 19 & 48,7 & 0 & 0,0 \\
\hline Líquido céfaloraquideo & 19 & 1 & 5,2 & 0 & 0,0 & 0 & 0,0 & 0 & 0,0 & 18 & 94,7 & 0 & 0,0 \\
\hline Biopsia pleural & 17 & 3 & 17,6 & 0 & 0,0 & 0 & 0,0 & 0 & 0,0 & 14 & 82,3 & 0 & 0,0 \\
\hline Otras biopsias & 14 & 0 & 0,0 & 1 & 7,1 & 0 & 0,0 & 0 & 0,0 & 13 & 92,8 & 0 & 0,0 \\
\hline Sangre y médula ósea & 14 & 0 & 0,0 & 0 & 0,0 & 0 & 0,0 & 0 & 0,0 & 5 & 35,7 & 9 & 64,3 \\
\hline Abcesos & 12 & 4 & 33,3 & 0 & 0,0 & 0 & 0,0 & 1 & 8,3 & 7 & 58,3 & 0 & 0,0 \\
\hline Orina & 12 & 3 & 25,0 & 4 & 33,3 & 0 & 0,0 & 0 & 0,0 & 5 & 41,7 & 0 & 0,0 \\
\hline Otras muestras * & 17 & 4 & 23,5 & 1 & 5,9 & 0 & 0,0 & 0 & 0,0 & 12 & 70,6 & 0 & 0,0 \\
\hline Total & 209 & 27 & 12,9 & 15 & 7,2 & 5 & 2,4 & 1 & 0,5 & 152 & 72,7 & 9 & 4,3 \\
\hline
\end{tabular}

ED+: examen directo positivo; ED-: examen directo negativo; EDnr: examen directo no realizado; C+: cultivo positivo; C-: cultivo negativo; Ccont: cultivo contaminado; Cnr: cultivo no realizado.

* Otras muestras incluyen 6 líquidos peritoneales, 5 líquidos pericárdicos, 5 muestras fecales y un líquido sinovial. 
Principales obstáculos que afectan

el desempeño de las redes

Los responsables de laboratorios centrales de tuberculosis de provincias recibieron un cuestionario acerca de los principales obstáculos que afectaban el desempeño de sus redes. Indicaron que las actividades más frecuentemente afectadas eran la realización de cultivos y la relectura de frotis para la evaluación de la calidad de baciloscopias. La escasez de personal entrenado y de adecuado equipamiento se señalaron como las principales causas que afectaban el desarrollo de las actividades mencionadas.

\section{Discusión}

Este trabajo describe los resultados de una encuesta transversal a los laboratorios del sector público que se llevó a cabo con el fin de determinar si la organización y funcionamiento de los laboratorios se adaptaba a las necesidades de implantación y expansión de la estrategia tratamiento acortado estrictamente supervisado impulsada por el PNCTB. Algunos de los datos recabados, como el volumen de pacientes sintomáticos respiratorios, examen microscópico directo y casos, se superponen con la información recolectada rutinariamente por la red nacional de laboratorios de tuberculosis. Sin embargo, en los últimos años, la disponibilidad de esta información en dos importantes jurisdicciones ha sido limitada, lo que ha comprometido el análisis nacional. Esto ha obligado a que estos datos deban ser recogidos en estudios especiales como el presente, en los que el esfuerzo del equipo de trabajo, permitió incrementar la cobertura, pero en perjuicio de la imposibilidad de realizar una evaluación sistemática y continua a nivel nacional. Además, dado que el período que cubre este trabajo es de cuatro meses, los datos no permiten reflejar las variaciones estacionales descritas para parámetros como el número de pacientes sintomáticos respiratorios o la tasa de positividad.

Los datos de este estudio están basados en las respuestas de los participantes a un cuestionario enviado por correo. En este tipo de metodología, la ausencia de un entrevistador en el momento de la recolección de los datos, reduce la posibilidad de recoger respuestas de mayor complejidad e impide la clarificación de aquellas preguntas que para el participante pudiesen resultar confusas, lo que puede conllevar a ciertas inexactitudes en los datos y/o a un aumento del porcentaje de norespuesta. No obstante, esta metodología ha permitido obtener información de los laboratorios que cubren alrededor del $90 \%$ de la población del país, cobertura que por razones logísticas (gran extensión del país) y económicas hubiese sido prácticamente imposible de lograr, utilizando otras metodologías.

De acuerdo a la densidad de servicios encontrada tanto globalmente como en el interior de las provincias, la oferta de centros de examen microscópico directo y cultivo se encuentran al nivel o por encima de los recomendados $1 / 100.000$ y 1/1.000.000 de habitantes, respectivamente 7 . Sin embargo, mientras que el volumen de muestras que un laboratorio de micobacteriología debería procesar para mantener destreza ha sido establecido en al menos 10 muestras/semana 7 para la lectura de baciloscopias y 20 muestras/ semana para laboratorios de cultivo 14 , en esta encuesta, $75 \%$ de los centros de microscopía procesaban < 10 muestras/semana y $48 \%$ de los centros de cultivo realizaban $<20$ cultivos/semana. Globalmente, estos porcentajes podrían ser mayores, si consideramos que, en base a nuestras estimaciones, la mayoría de los centros "no participantes" procesaban $<10$ baciloscopías / semana y $<20$ cultivos/semana. Claramente, en varios laboratorios el mantenimiento de la destreza de su personal podría verse dificultado por el procesamiento de pocas muestras. Sin embargo, la concentración de centros de diagnóstico en pocos laboratorios no parece ser una estrategia adecuada, ya que la geografía de Argentina se caracteriza por la existencia de algunas áreas con escasa población y en las que las comunicaciones podrían ser insuficientes. Durante el año 2003, alrededor del 70\% de los exámenes microscópicos directos se realizaron en centros que participaron en el programa de evaluación de la calidad de microscopía; es imprescindible el incremento de los esfuerzos para mejorar la cobertura de este programa, a fin de asegurar la calidad de todos los diagnósticos microbiológicos. Especial énfasis debería realizarse en laboratorios de baja carga de trabajo en los que es más dificultoso el mantenimiento de la calidad. Por otro lado, es importante recalcar que la gran oferta de centros contribuirá a afrontar exitosamente la creciente demanda de diagnóstico que es esperable cuando la estrategia tratamiento acortado estrictamente supervisado está en proceso de expansión, como en Argentina.

El examen microscópico directo para la detección de bacilos ácidos-alcohol resistentes no es una técnica totalmente reproducible aun en las mejores condiciones de relectura. En estudios realizados por lectores expertos 15,16, la reproducibilidad varió entre el $93 \%$ y $96 \%$. Por esta razón, una concordancia global del 98,5\% puede ser considerada como muy buena. Resultados similares fueron obtenidos en otros países de la 
región 17,18. La observación de que 30\% de los resultados falsos positivos correspondían a extendidos con cristales de fucsina o con insuficiente decoloración indica que no sólo es necesario reentrenar acerca de la capacidad de distinguir bacilos, sino también del método de coloración.

La oferta de servicios de pruebas de sensibilidad a quimioterápicos resultó superior a la recomendada de 1/10.000.000 habitantes. La confiabilidad de la identificación de bacilos multirresistentes es esencial para el control de la tuberculosis multirresistente. Desde 1999, la red nacional de laboratorios de tuberculosis cuenta con un programa de aseguramiento de calidad de pruebas de sensibilidad a quimioterápicos, coordinado por el laboratorio del INEI. Éste consiste en la remisión de paneles de cultivos de Mycobacterium tuberculosis a los laboratorios, a fin de que sean evaluadas su sensibilidad a Isoniacida, Rifampicina, Estreptomicina y Etambutol. Dichos paneles, preparados por los laboratorios supranacionales, consisten en aislamientos de M. tuberculosis clasificados como sensibles o resistentes, usando el resultado de consenso de los laboratorios supranacionales. En el año 2002, los resultados del programa de competencia 19 evidenciaron una alta precisión para Isoniacida y Rifampicina, alcanzándose, en la mayoría de los laboratorios de la red, el nivel de eficiencia requerido por OMS 20. Contrariamente, los resultados para Etambutol y Estreptomicina tuvieron mayor dispersión y menor confiabilidad; tres laboratorios tuvieron resultados de eficiencia para la sensibilidad a Isoniacida y/o Rifampicina, claramente por debajo de los niveles de considerados aceptables 20 , lo que dio lugar a acciones correctivas.

Aún cuando la Unión Internacional contra la Tuberculosis y Enfermedades Respiratorias recomienda estudiar un promedio de 10 pacientes sintomáticos respiratorios para identificar un caso positivo 21 , se considera que, en general, tasa de positividad está influenciada tanto por la incidencia de tuberculosis, como por la estrategia de detección de casos aplicada en cada lugar. Así, Colombia, con similar tasa de notificación de casos de tuberculosis que Argentina, reportó para el año 200222 una tasa de positividad de 28:1, muy próxima a la encontrada en este estudio (26:1). Sin embargo, el rango de la tasa de positividad encontrada en las distintas provincias fue amplio (16:1-147:1), no correlacionándose con la tasa de notificación de casos, posiblemente debido a que la mayoría de las jurisdicciones con alta carga de tuberculosis tienen muy buena disponibilidad de centros de diagnóstico (buena oferta) y además, en estas provincias se aplican políticas de búsqueda de casos que alientan la investigación de pacientes con síntomas respiratorios a través de visitas periódicas a sus hogares de agentes de salud (búsqueda de casos activa).

El número óptimo de muestras de esputo necesario para establecer diagnóstico ha sido examinado en numerosos estudios. Una reciente revisión 23 muestra que en promedio la muestra inicial es positiva en $83-87 \%$ de los pacientes diagnosticados de tuberculosis por baciloscopia, la segunda adiciona entre $10-12 \%$, mientras la tercera aporta sólo 3-5\%. Por ello, las recomendaciones internacionales 23 sugieren que todo paciente sospechoso de tuberculosis, debe tener al menos dos muestras de esputo analizadas por microscopia. En algunos lugares, por razones logísticas y prácticas, una tercera muestra puede ser de utilidad. En Argentina, la política de localización de casos recomendada está basada en la recolección de al menos dos muestras de esputo/paciente 24 . Sin embargo, este estudio muestra que el número de muestras/paciente es relativamente bajo $(1,6)$, igual al informado en Colombia $(1,6) 22$, aún cuando en este país la política de búsqueda está basada en la recolección de tres muestras/paciente. Estos datos acentúan la necesidad de incrementar los esfuerzos para la recolección de la segunda muestra; en este sentido la experiencia en La Habana (Cuba) muestra que la aplicación de un plan de reorientación de su programa logró incrementar este indicador de 1,7 en 1995 a 1,9 en 199925 .

Los estándares internacionales para el diagnóstico de tuberculosis 22 recomiendan monitorear la respuesta al tratamiento mediante microscopía de esputo al menos al final de la primera fase, a los cinco meses y al final de tratamiento. En este estudio no se contempló la recolección del volumen de muestras procesadas para control de tratamiento. No obstante, los datos de la supervisión directa a servicios de salud de 17 jurisdicciones mostraban, en el año 2003, que sólo en el $38 \%$ de los registros de los pacientes tuberculosos en tratamiento se consignaba la solicitud de baciloscopias de control de tratamiento 26 , evidenciándose la necesidad de intensificar las actividades de capacitación y supervisión del equipo de salud.

Todos los laboratorios de cultivo participantes empleaban medio de cultivo sólido a base de huevo, mayoritariamente de origen "casero". Sólo $12 \%$ de los laboratorios empleaban sistemas con medios líquidos. En otros estudios, realizados en Europa 27,28 y Estados Unidos 29, la mayoría de los servicios empleaban estos medios y métodos para facilitar un diagnóstico temprano y certero. En Argentina, la escasez de recursos y presupuestos adecuados hacen necesario continuar con el empleo de sistemas de cultivo tradicionales. Sin embargo, a pesar de la baja can- 
tidad de sistemas basados en medios líquidos, este estudio demuestra que estos sistemas están ubicados en ciudades que concentran la mayoría de los pacientes infectados con VIH, en los que el uso de métodos más rápidos y sensibles es una prioridad.

Este trabajo mostró que en el 25\% de los laboratorios de cultivo su personal no utiliza o utiliza inadecuadamente barreras respiratorias. La prevalencia de tuberculosis entre el personal de laboratorio durante los últimos 10 años (3,96\%) fue alta en relación a población general. Nuestros datos son similares a los encontrados por DeRiemer et al. 30 en un reciente estudio realizado en Brasil. Otras investigaciones han reportado que el riesgo de padecer tuberculosis entre los trabajadores de laboratorio es 100 veces mayor que el de la población general ${ }^{31}$. Aún cuando, este estudio no fue diseñado para determinar si la historia de tuberculosis fue el resultado de la exposición ocupacional, este trabajo refuerza la importancia de proveer a los trabajadores de laboratorio de cultivo de adecuada protección respiratoria y de una evaluación médica anual.

Este trabajo evidenció que las muestras de lavado broncoalveolar con examen microscópico directo positivo no eran sistemáticamente cultivadas, a pesar de los estudios que describen la aparición de resultados falsamente positivos al examen microscópico directo a consecuencia de contaminación cruzada con micobacterias durante los procedimientos broncoscópicos; este fenómeno se ha atribuido a la presencia de bacilos muertos (que por tanto no desarrollan al cultivo) que persisten en el broncoscopio después de los procesos de desinfección y lavado, y que pueden dar lugar a un resultado positivo por examen microscópico directo en la muestra subsiguiente 32,33 .

Generalmente, en los sitios extrapulmonares existen pocos bacilos, por lo que la detección por examen microscópico directo es poco frecuente 23. Así, en nuestro estudio la mayoría de las muestras extrapulmonares resultaron positivas sólo por cultivo; por tanto, en pacientes con sospecha de tuberculosis extrapulmonar es obligatorio el uso del cultivo.

Las técnicas de cultivo incrementan el número de casos detectados, y permiten diagnosticarlos más tempranamente, con frecuencia antes de que éstos se vuelvan más infecciosos 11. Sin embargo, el empleo del cultivo para todos los pacientes con microscopía negativa resultaría una estrategia muy costosa e insustancial, teniendo en cuenta que se trata de un recurso no tan fácilmente disponible.

Cuando el cultivo se realiza apropiadamente, el mismo puede identificar hasta $30-40 \%$ de los casos bacteriológicamente confirmados 23,34. En este trabajo, el porcentaje de casos "sólo cultivo positivo" fue de $18,9 \%$, con un rango de 0 a $42 \%$ entre las 24 provincias. Aquellas jurisdicciones que cultivaron hasta $20-30 \%$ de las muestras respiratorias obtenidas identificaron hasta un 20-30\% de sus casos sólo por cultivo, mientras que aquellas en las que la mayoría de los casos eran examen directo-positivo sólo cultivaban $<5 \%$ de sus muestras. Esto indicaría la necesidad de incrementar el número de esputos cultivados, para así lograr aumentar el diagnóstico de pacientes examen directo-negativo, que como ya se ha evidenciado pueden ser una fuente de transmisión significativa en la comunidad 35 . Por otro lado, aún cuando la mayoría de los laboratorios indicaron que utilizaban el cultivo, de acuerdo a las normas impartidas por la red nacional de laboratorios de tuberculosis, resulta poco claro el motivo por el cual existe tanta variabilidad en el porcentaje de muestras que se cultivan. Es posible que en muchos casos la información recibida por los bacteriólogos para determinar la necesidad del cultivo sea incompleta, lo que determina una subutilización del recurso. Otras variables relacionadas con la disponibilidad de servicios de cultivo no resultaron asociadas al aporte del cultivo, posiblemente, debido a la elevada oferta de servicios en las jurisdicciones.

La coinfección con VIH, así como la edad de los pacientes, son factores determinantes de las características clínicas y bacteriológicas de la enfermedad. Así, entre los pacientes coinfectados 36 o en < 15 años 11,23 la proporción de casos sólo cultivo positivo se incrementa. En este trabajo la información acerca de la condición de infección por VIH no fue recolectada, ya que no es un dato con el que cuenten de rutina los bacteriólogos y tampoco es confiable en los registros de casos notificados al PNCTB. En el año 2003, en Argentina no existía una política de evaluación sistemática de la infección por VIH entre los casos de tuberculosis. Por tanto, no se pudo estudiar su posible efecto confundente en la evidenciada asociación entre la proporción de muestras respiratorias cultivadas y el nivel de detección de casos por cultivo encontrado en las distintas provincias. Sin embargo, aún cuando se excluyó la información de dos jurisdicciones que concentran el 75\% de los casos de SIDA 37, el porcentaje de las muestras respiratorias que se cultivan siguió estando independientemente asociado al aporte del cultivo. Tampoco se estudió la posible influencia de la edad sobre el aporte del cultivo, aunque es poco probable que esta variable tenga influencia ya que según los registros del PNCTB, el porcentaje de pacientes pediátricos con confirmación bacteriológica es 
bajo y muy similar entre las distintas jurisdicciones (rango: 2,5-5\%) 1.

Los responsables de los laboratorios centrales de tuberculosis de provincias identificaron la baja cobertura del control de calidad de microscopía y la ausencia de adecuado equipamiento como los principales obstáculos que afectaron el funcionamiento de sus redes. Teniendo en cuenta estos hallazgos, durante el año 2004 y 2005, se destinaron fondos del tesoro nacional para la compra de equipamiento de laboratorio ${ }^{38}$. Se puso énfasis en incrementar la capacidad diagnóstica de los laboratorios centrales de tuberculosis de provincias, debido a que funcionan como centros de referencia primaria de cada región y son responsables de una parte sustancial del programa de calidad externa de examen microscópico directo. Además, se han distribuido cabinas de seguridad biológica a los laboratorios con alta carga de cultivos. También, y a fin de incrementar la cobertura del programa de calidad de microscopía, los laboratorios centrales han recibido microscopios y se ha desarrollado

\section{Resumen}

Con el objetivo de evaluar recursos, prácticas y medidas de bioseguridad utilizados en el diagnóstico bacteriológico de tuberculosis y determinar la utilidad y alcance de las técnicas empleadas, se llevó adelante una encuesta transversal a laboratorios del sector público de Argentina utilizando un cuestionario estructurado. El análisis de los resultados mostró que la densidad de centros de microscopía y cultivo se encuentra al nivel o encima de lo recomendado por Organización Mundial de la Salud. El programa de control de calidad de microscopía cubre mayoritariamente los laboratorios con alta carga de trabajo. El promedio de baciloscopías/paciente fue bajo (1,6). El 25\% de los laboratorios de cultivo empleaban protección respiratoria inadecuada. El aporte del cultivo a la confirmación de casos pulmonares en las jurisdicciones estuvo asociado a la proporción de muestras cultivadas. Globalmente, el porcentaje de pacientes pulmonares baciloscopía-negativa que fue confirmado bacteriológicamente fue de 18.9\%; mientras que la mayoría de los casos extrapulmonares se confirmó sólo por cultivo (71,3\%). Es prioritario aún el incremento del número de esputos estudiados por baciloscopía y cultivo, así como aumentar la cobertura de programa de control de calidad de microscopía; es fundamental proveer al personal de laboratorio de adecuada protección respiratoria y evaluación médica regular.

Tuberculosis; Técnicas y Procedimientos de Laboratorio; Recolección de Datos un programa de capacitación focalizado en aspectos técnicos, operacionales y en el sistema de calidad.

En conclusión, los resultados de este estudio muestran que en Argentina es prioritario aún el incremento del número de muestras y pacientes estudiados por microscopía y cultivo, así como un aumento en la cobertura del programa de evaluación de la calidad de microscopía. Estos objetivos parecen hoy más accesibles ya que ciertos prerrequisitos, como la disponibilidad de servicios de microscopía y cultivo y la provisión de equipamiento adecuado para incrementar las capacidades diagnósticas de los laboratorios, están garantizados. Con el propósito de lograr el control y eliminación de la tuberculosis, es imprescindible que los laboratorios de micobacterias sean utilizados adecuadamente para el diagnóstico, seguimiento del tratamiento y vigilancia epidemiológica. La evaluación de la disponibilidad y funcionamiento de los servicios de laboratorios son de importancia capital para los PNCTB.

\section{Colaboradores}

M. D. Sequeira y M. S. Imaz realizaron el diseño del estudio, el protocolo de trabajo, la puesta en marcha, el análisis de datos y resultados. Los miembros de los Laboratorios Centrales de Tuberculosis de las provincias participaron en la revisión del protocolo de trabajo y llevaron adelante el trabajo de campo.

\section{Otros miembros de la Red de Laboratorios Provinciales de Tuberculosis en Argentina}

A. Aguirre, C. Alexandre, L. Alfonso, R. Arrieta, D. Ballester, A. Bayesti, D. Berry, G. Bichara, N. Bitar, N. Blázquez, J. Bolpe, R. D. Calleja, V. Carrió, D. Chanampe, M. Cisneros, S. I. Contreras, C. Cosiansi, R. Costa, S. Dalmaso, Y. Echagüe, C. Elorza, A. Etchart, M. Fantín, E. Fernández, S. Gagliardi, V. Gandini, V. García, M. I. Gilli, F. Gómez, E. Gravina, C. Gronda, A. Guerra, M. Gunia, A. Hermida, T. Hernández, J. Herrera, M. Hoffman, J. Lerena, L. Mariñaski, M. Massain, N. Morcillo, S. Muñoz, G. Natali, M. T. Pacce, R. Pereyra, J. Prados, L. Psenda, M. Rivas, A. Romite, A. Rosseti, H. Rouselle, A. Rybko, A. Sologuren, A. Spoti, A. Tayre, A. Togneri, G. Torres, L. Yeschke, A. Zapata.

\section{Agradecimientos}

A Omar Latini por su contribución en la preparación de este manuscrito y a Isabel Kantor por su revisión critica. 


\section{Referencias}

1. Instituto Nacional de Enfermedades Respiratorias Dr. Emilio Coni. Situación de la tuberculosis. República Argentina, 2003. Santa Fe: Instituto Nacional de Enfermedades Respiratorias Dr. Emilio Coni; 2004.

2. World Health Organization. WHO report: global tuberculosis control-surveillance, planning, financing. Geneva: World Health Organization; 2004.

3. Instituto Nacional de Enfermedades Respiratorias Dr. Emilio Coni. Evaluación de tratamiento de los casos de tuberculosis pulmonar examen directo positivo. República Argentina, 2002. Santa Fe: Instituto Nacional de Enfermedades Respiratorias Dr. Emilio Coni; 2004.

4. World Health Organization. An expanded DOTS framework for effective tuberculosis control. Geneva: World Health Organization; 2002.

5. Enarson DA. The international union against tuberculosis and lung disease model national tuberculosis programmes. Tuber Lung Dis 1995; 76: 95-9.

6. World Health Organization. WHO report on global tuberculosis epidemic 1998. Geneva: World Health Organization; 1998.

7. World Health Organization. Laboratory services in tuberculosis control. Part I: organization and management. Geneva: World Health Organization; 1998.

8. Kirkwood B. Essentials of medical statistics. Oxford: Blackwell Science; 1998.

9. Instituto Nacional de Enfermedades Respiratorias Dr. Emilio Coni. Manual de microscopía. Normas técnicas. Santa Fe: Instituto Nacional de Enfermedades Respiratorias Dr. Emilio Coni; 2000.

10. Centro Panamericano de Zoonosis. Manual de normas y procedimientos técnicos para la bacteriología de la tuberculosis. Parte II. El cultivo del Mycobacterium tuberculosis. Nota técnica 27. Martínez: Organización Panamericana de la Salud; 1985.

11. World Health Organization. Laboratory services in tuberculosis control. Part III: culture. Geneva: World Health Organization; 1998.

12. Kusznierz GF, Latini OA, Sequeira MD. Quality assessment of smear microscopy for acid-fast bacilli in the Argentine tuberculosis laboratory network, 1983-2001. Int J Tuberc Lung Dis 2004; 8:1234-41.

13. Bordabossana D, Texidor CA, Domenech MC, Volpe E, Pasqualini E. Nuevo método sencillo y económico para facilitar el cultivo del bacilo tuberculoso. Rev Argent Tuberc Enferm Pulmonares 1977; 3/4:15-8.

14. Level of laboratory services for mycobacterial diseases: American Thoracic Society. Medical Section of the American Lung Association. Am Rev Respir Dis 1983; 128:213.

15. Pollak L, Urbanczic R. La relación entre la calidad de las muestras y la positividad en microscopia. Boletín Informativo del Instituto Nacional de Tuberculosis (Caracas) 1969; 2:5-8.

16. Daniel TM. Toman's tuberculosis. Case detection, treatment and monitoring: questions and answers. $2^{\text {nd }}$ Ed. Geneva: World Health Organization; 2004.
17. Franco C. Calidad del examen directo de esputo en la Red de Laboratorios de Bacteriología de Tuberculosis de Colombia. Bol Oficina Sanit Panam 1993; 115:103-9.

18. Valenzuela M, Ponce J, Lepe R, Piffardi S, Velasco M, García P. Supervisión técnica indirecta de baciloscopias. Bol Inf COLABAT 1988; 4:6-9.

19. Instituto Nacional de Enfermedades Respiratorias Dr. Emilio Conir. Plan de actividades 2006 de la Red Nacional de Laboratorios de Tuberculosis. Santa Fe: Instituto Nacional de Enfermedades Respiratorias Dr. Emilio Conir; 2005.

20. Laszlo A, Rahman M, Espinal M, Raviglione M; WHO/IUATLD Network of Supranational Reference Laboratories. Quality assurance programme for drug susceptibility testing of Mycobacterium tuberculosis in the WHO/IUATLD Supranational Reference Laboratory Network: five rounds of proficiency testing, 1994-1998. Int J Tuberc Lung Dis 2002; 6:748-56.

21. International Union Against Tuberculosis and Lung Diseases. Technical guide for sputum examination for tuberculosis by direct smear microscopy. 3rd Ed. Paris: International Union Against Tuberculosis and Lung Diseases; 1978.

22. Chaparro PE, García I, Guerrero MI, León CI. Situation of tuberculosis in Colombia, 2002. Biomedica 2004; 24(1 Suppl):102-14.

23. Tuberculosis Coalition for Technical Assistance. International standards for tuberculosis care (ISTC). The Hague: Tuberculosis Coalition for Technical Assistance; 2006.

24. Instituto Nacional de Enfermedades Respiratorias Dr. Emilio Coni. Normas técnicas del Programa Nacional de Control de la TB. Santa Fe: Instituto Nacional de Enfermedades Respiratorias Dr. Emilio Coni; 2002.

25. Sevy-Court JI, Peláez-Sánchez O, Arteaga-Yero AL, Aramas-Pérez L, Borroto-Gutiérrez S, GonzálezOchoa CE. Tuberculosis en la ciudad de la Habana 1995-1999. Rev Saúde Pública 2003; 37:326-32.

26. Instituto Nacional de Enfermedades Respiratorias Dr. Emilio Coni. Informe final y conclusiones de XXXVII Reunión del Consejo Confederal de Control de Tuberculosis de Argentina. Santa Fe: Instituto Nacional de Enfermedades Respiratorias Dr. Emilio Conir; 2006.

27. Piersimoni C, Mandler F, Marchetti D, Molinari GL, Riva R, Tortoli E, et al. Mycobacterial testing in hospital laboratories: results from a questionnaire survey in Italy. Clin Microbiol Infect 2004; 10 : 1014-7.

28. Drobniewski FA, Watt B, Smith EG, Magee JG, Williams R, Holder J, et al. A national audit of the laboratory diagnosis of tuberculosis and other mycobacterial diseases within the United Kingdom. J Clin Pathol 1999; 52:334-7.

29. Denniston MM, Bird BR, Kelley KA. Contrast of survey results between state and a cohort of nonstate mycobacteriology laboratories: changes in laboratory practices. J Clin Microbiol 1997; 35:422-6. 
30. DeRiemer K, Martins-Moreira F, Werneck-Barreto AM, Ueleres-Braga J. Survey of mycobacteriology laboratory practices in an urban area with hyperendemic pulmonary tuberculosis. Int J Tuberc Lung Dis 2000; 4:776-83.

31. Collins CH, Grange JM. Tuberculosis acquired in laboratories and necropsy rooms. Commun Dis Public Health 1999; 2:161-7.

32. Gubler JG, Salfinger M, von Graevenitz A. Pseudoepidemic of nontuberculous mycobacteria due to a contaminated bronchoscope cleaning machine. Report of an outbreak and review of the literature. Chest 1992; 101:1245-9.

33. Reeves DS, Brown NM. Mycobacterial contamination of fibreoptic bronchoscopes. J Hosp Infect 1995; 30:531-6.

34. Foulds J, O'Brien R. New tools for the diagnosis of tuberculosis: the perspective of developing countries. Int J Tuberc Lung Dis 1998; 2:778-83.

35. Behr MA, Warren SA, Salamon H, Hopewell PC, Ponce-de-Leon A, Daley CL, et al. Transmission of tuberculosis from patients smear-negative for acid-fast bacilli. Lancet 1999; 353:444-9.
36. Liberato IRO, Albuquerque MFPM, Campelo ARL, Melo HRL. Characteristics of pulmonary tuberculosis in HIV seropositive and seronegative patients in Northeastern region of Brazil. Rev Soc Bras Med Trop 2004; 37:46-50.

37. Ministerio de Salud y Ambiente de la Nación. Boletín sobre VIH-SIDA en la Argentina. Año XI. no. 23. Buenos Aires: Ministerio de Salud y Ambiente de la Nación; 2004.

38. Instituto Nacional de Enfermedades Respiratorias Dr. Emilio Coni. Cumplimiento de objetivos previstos. Programa VIGIA, 2000-2005. Santa Fe: Instituto Nacional de Enfermedades Respiratorias Dr. Emilio Coni; 2006.

39. Kubica GP, Dye WE, Cohn ML, Middlebrook G. Sputum digestion and decontamination with $\mathrm{N}$ acetyl-L-cysteine-sodium hydroxide for culture of mycobacteria. Am Rev Respir Dis 1963; 87:775-9.

40. Tacquet A, Tison F. New technic of isolation of mycobacteria by sodium laurylsulfate. Ann Inst Pasteur (Paris) 1961; 100:676-80.

Recibido el 24/Ago/2005

Versión final presentada el 24/Ago/2006

Aprobado el 19/Sep/2006 